\title{
Frequency Tracking of A Prototype Elliptical RF Cavity
}

\author{
Rajdip Das \\ Department of Electronics and Communication \\ Engineering \\ Supreme Knowledge Foundation Group of Institutions \\ 1, Khan Road Mankundu, Hooghly, 712123, \\ West Bengal, India
}

\begin{abstract}
Remarkable development with the use of microwave cavity has occurred during the last two to three decades, particularly in portable correspondence, TV transmission, modern/local applications, satellite correspondence, telemetry, RADAR/navigational guides, and so forth. This quick improvement inside the microwave which of computerized advances has synergized, bringing about further quickened rate. The paper reports primarily on the frequency tracking system probably be coupled with radio frequency $(\mathrm{RF})$ cavity. For the creating and testing of the tracking system, we have used phase detectors and loop filters throughout. The quality of the tracking system and thus the underlying scanning results were seen to help the realistic tests.
\end{abstract}

Keywords-RF cavity, phase detector, loop filter, frequency tracking.

\section{INTRODUCTION}

In many applications and systems, most of the information carried in signals is contained in their amplitude and their frequency content. Typically, these characteristics are examined from two complementary viewpoints: the time and frequency domains. These two domains are alternative perspectives on the power of a signal. The former is particularly suited for investigating its temporal evolution, whereas the latter is most relevant when inspecting its repartition among the frequencies. The same is true for an RF cavity resonator.

A microwave cavity or radio frequency (RF) cavity [1] is a special form of resonator [2], consisting of a closed (or largely closed) metal structure that confines electromagnetic fields within the microwave region of the spectrum. The basic structure is either hollow or filled up with dielectric material. At resonance, a resonant electromagnetic cavity is usually represented as a parallel RLC circuit. For typical values of R, $\mathrm{L}$, and $\mathrm{C}$ the RF cavity is considered to be a Bandpass filter. The ratio between the energy stored in the reactive components and the losses in the resistive component at resonance gives a measurement of the resonator's quality factor, Q. Generally the RF cavity has Q in the order of $10^{\wedge} 6$ which is much higher than the corresponding $Q$ values of the rest of the system elements. It is this Q only which dictates the response of the system to the input modulation techniques as compared to the other electronic components present in the system. Hence at the resonant frequency of the RF cavity, we get the maximum output. A shift from the resonant frequency due to variation in its structure results in a decrease/increase in the amplitude.

\author{
Soumen Khatua \\ Professor Department of Electronics and Communication \\ Engineering \\ Supreme Knowledge Foundation Group of Institutions \\ 1, Khan Road Mankundu, Hooghly, 712123, \\ West Bengal, India
}

The goal of this research is to design a frequency tracking system to detect the variation in the amplitude due to the presence of dynamic and static variations in the structure which is causing a shift in the resonance frequency of the cavity and to correct the same.

The paper is organized as follows: The designing of a frequency tracking system is presented in Section III, and experimental results in Section IV. Finally, the conclusion and future works are discussed in Section V.

\section{BACKGROUND SURVEY}

\section{A. Radio Frequency Cavity Characterisation}

At resonance, a resonant electromagnetic cavity is usually represented as a parallel RLC circuit, whose shunt impedance (resistance), $\mathrm{R}$, is determined from its losses at resonance and whose reactive values are determined from the energy stored at resonance. The ratio between the energy stored in the reactive components and the losses in the resistive component at resonance gives a measurement of the resonator's quality factor, Q [3]. Intuitively, this Q-factor will influence the response of a resonant structure to amplitude modulations. To see this link a bit more let us consider the following:

1) Amplitude modulation [6],[8] of a carrier frequency generates a frequency spectrum equivalent to the spectrum of the modulating signal centered about the carrier frequency. Thus, let us consider the scenario that a resonant cavity is being driven at its resonance frequency, but that the amplitude of the driving signal is being modulated.

2) As the frequency of the amplitude modulating signal increases the sidebands of the resultant modulated spectrum will be pushed outside of the resonator's bandwidth and will thus suffer a dampening effect due to the resonator's frequency selectivity.

Now we mathematically characterize the resonator's response to amplitude modulations, we assume that the resonant cavity can be modeled as a parallel RLC circuit within the quality factor bandwidth. We begin by determining the transfer function relating the output voltage to the driving current of a parallel RLC circuit. The transfer function turns out to be the expression for the impedance of the circuit as a function of frequency, given as. Now we mathematically characterize the resonator's response to amplitude modulations. The expression for the impedance of the circuit as a function of frequency, given as, 


$$
Z(f)=\frac{V(f)}{I(f)}=\frac{j \frac{2 \pi f}{C}}{\frac{1}{L C}+j \frac{2 \pi f}{R C}-(2 \pi f)^{2}}
$$

Now, when we apply amplitude modulation to the RF drive signal, we are applying a signal with a certain frequency spectrum. This frequency spectrum is then applied to the resonator which acts as a filter. When we then detect the voltage (or phase) we are demodulating by shifting the filtered spectrum down to a baseband for which DC corresponds to the non-modulated carrier RF frequency. This demodulation process is mathematically equivalent to multiplying the filtered signal by a sinusoid whose frequency is equal to the resonant frequency of the parallel RLC circuit. In the frequency domain, the demodulation process is described mathematically with the use of Fourier transforms. The modulation theorem is given as,

$s(t) \cdot \cos \left(2 \pi f_{R} t\right) \Leftrightarrow \frac{1}{2} S\left(f-f_{R}\right)+\frac{1}{2} S\left(f+f_{R}\right)$

Now, we will find out the impulse response of the system by giving a delta function as the input. Thus the output becomes,

$$
Z(f) \otimes \mathfrak{S}\left\{\cos \left(2 \pi f_{R} t\right)\right\}=\frac{1}{2} Z\left(f-f_{R}\right)+\frac{1}{2} Z\left(f+f_{R}\right)
$$

Using the above equation in equation (1) and applying Laplace transform to it we get the final expression as, $Z(s)=$

$$
\begin{array}{r}
\frac{s^{3} L C R+s^{2} L+s\left(R+4 \pi^{2} f_{R}^{2} L C R\right)+4 \pi^{2} f_{R}^{2} L}{s^{4}(L C R)^{2}+s^{3} 2 L^{2} C R+s^{2}\left[L^{2}+2 L C R^{2}+8 \cdot\left(\pi f_{R} L C R\right)^{2}\right]+s\left[2 R L+8 C R\left(\pi f_{R} L\right)^{2}\right]+\ldots} \\
{\left[R^{2}+\left(4 \pi^{2} f_{R}^{2} L C R\right)^{2}-8 L C\left(\pi f_{R} R\right)^{2}+\left(2 \pi f_{R} L\right)^{2}\right]}
\end{array}
$$

In the expression, the pair of complex conjugate zeros and the pair of complex conjugate poles composes the BPF. Fig. 1 below depicts the frequency spectrum of an RF cavity.

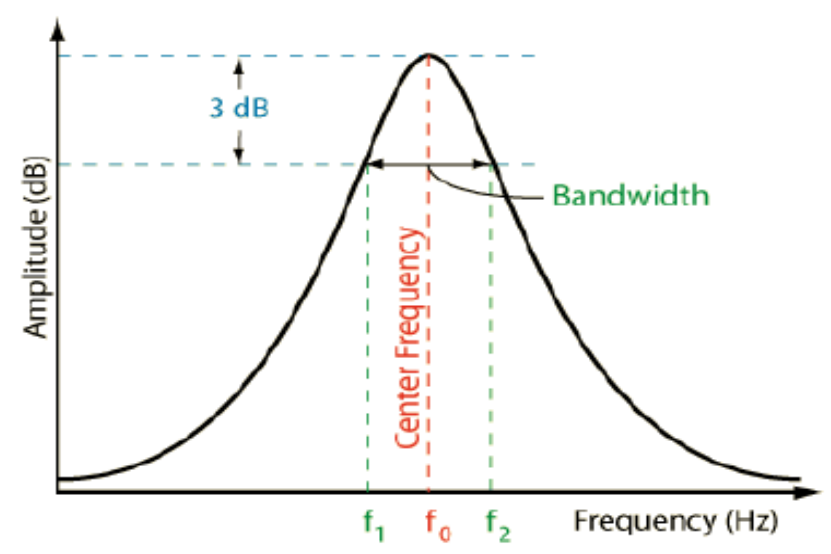

Fig 1. The frequency spectrum of an RF Cavity

Here, $f_{0}$ refers to the resonant frequency of the cavity, and $f_{1}$ and $\mathrm{f}_{2}$ refer to the $3 \mathrm{~dB}$ frequency. The bandwidth of the cavity is given by $f_{1}-f_{2}$.

Since the cavity is approximated to be a parallel RLC circuit it is necessary to derive the relation between the $\mathrm{Q}-\mathrm{a}$ factor of the circuit and the bandwidth of the circuit. A parallel RLC circuit has been shown below and the desired derivation has been carried out thereafter. Here the impedance seen by the current source is:

$$
Z_{\| /}=\frac{j \omega L}{\left(1-\omega^{2} L C\right)+\frac{j \omega L}{R}}
$$

At the resonance frequency $\left(1-\mathrm{w}^{2} \mathrm{LC}\right)=0$, the impedance is seen by the source is purely resistive. The parallel combination of the capacitor and the inductor acts as an open circuit. Therefore at the resonance, the total current flow through the resistor. The current flowing through the resistor is given by:

$$
\begin{aligned}
I_{R} & =I_{S} \frac{\frac{1}{Z_{R}}}{\frac{1}{Z_{R}}+\frac{1}{Z_{C}}+\frac{1}{Z_{I}}} \\
& =I_{S} \frac{j(\omega I}{\left(R-\omega^{2} L(R)+j \omega L\right.}
\end{aligned}
$$

Where Is refers to the current source. And the transfer function becomes:

$$
|H(\omega)|=\left|\frac{I_{R}}{I_{S}}\right|=\frac{\omega L}{\sqrt{\left(R-\omega^{2} L C R\right)^{2}+(\omega L)^{2}}}
$$

Taking the value of an inductor, $\mathrm{L}=47 \mathrm{mH}$ and capacitor, $\mathrm{C}$ $=47 \mathrm{uF}$ the transfer function was plotted for various values of $\mathrm{R}$ which is shown in Fig 2. The half-power frequencies are found out by letting $|\mathrm{H}(\mathrm{w})|=(1 / 2)^{\wedge}\left({ }^{1 / 2}\right)$ i.e.

$$
\frac{1}{\sqrt{2}}=\frac{\omega L}{\sqrt{\left(R-\omega^{2} L C R\right)^{2}+(\omega L)^{2}}}
$$

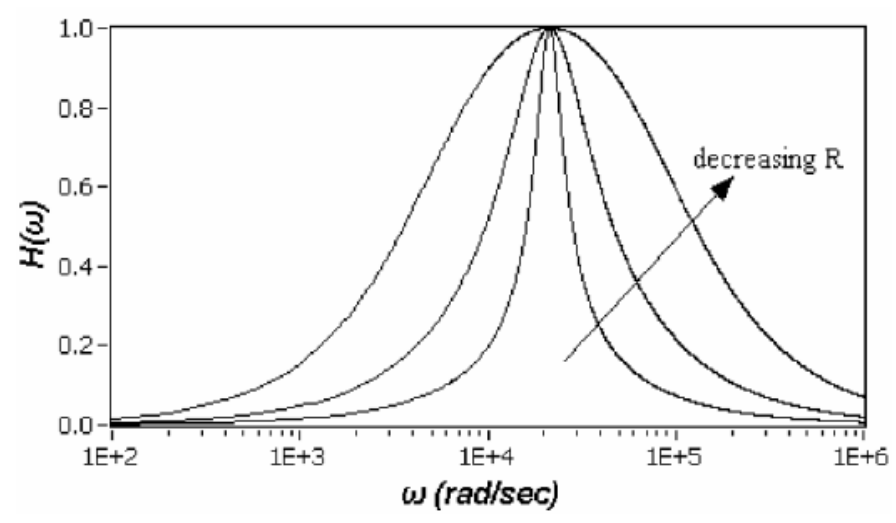

Fig 2. A plot of a transfer function for various values of $\mathrm{R}, \mathrm{L}$, and $\mathrm{C}$

By solving the above equation we get the two half-power frequencies as follows:

$$
\begin{aligned}
& \omega_{1}=-\frac{1}{2 R C}+\sqrt{\left(\frac{1}{2 R C}\right)^{2}+\frac{1}{\omega_{0}^{2}}} \\
& \omega_{2}=\frac{1}{2 R C}+\sqrt{\left(\frac{1}{2 R C}\right)^{2}+\frac{1}{\omega_{0}^{2}}}
\end{aligned}
$$

And the bandwidth for the parallel RLC circuit is given as:

$$
B_{P}=\omega_{2}-\omega_{1}=\frac{1}{R C}
$$

Now, the $\mathrm{Q}$ - factor is given by: 


$$
Q=\frac{\omega_{0}}{B_{P}}=\omega_{0} R C=\frac{R}{\omega_{0} L}
$$

Now, when a compression the or short - time vibrations are applied to the structure the center of the BPF shifts either to the right or left of the actual center i.e. $f_{0}$. This shift in the frequency would decrease the output signal's amplitude. This property can be taken into an advantage when considering the task at hand. Peak detectors can be used to detect the change in the amplitude and using this frequency tracking would be carried out.

\section{B. RF Peak Detectors}

There are various ways to detect the peak of an RF signal. The other possible designs of peak detectors are as follows:

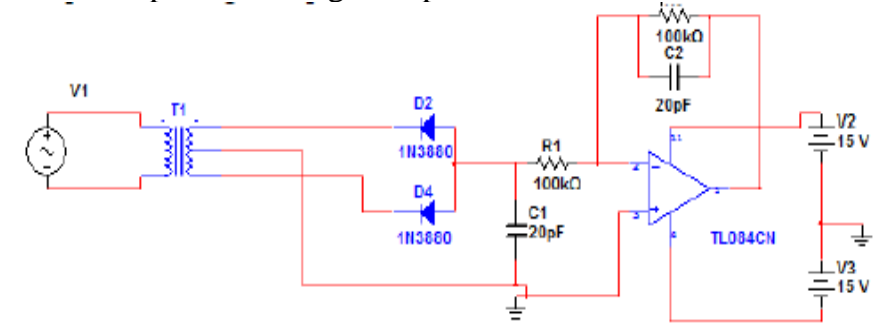

Fig 3. Simple peak detector

The detector shown in Fig 3. was simulated in multisim. Instead of using an ideal 1:1 transformer, the transformer with the ratio 4:1 was chosen. For various values of the input signal, the peak was measured. Due to the presence of $4: 1$ transformer, the output is not always the exact input value. From the measured output, it is observed that there is a linear relationship between the input and the output values. From Fig 4. we can figure out that the working of this peak detector greatly relies on the diodes. Here, $\mathrm{V}_{\text {dee }}$ refers to the output of the RF cavity. It is highly nonlinear for the input in the range below of 0.2 volts.

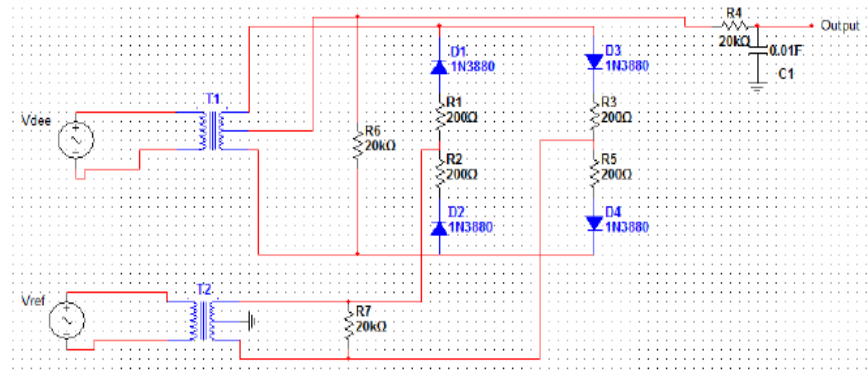

Fig 4. Coherent detection

The detector B is an improvement over detector A but it has a drawback that its output is dependent on the phase of the RF cavity. For a better understanding of the drawbacks of a peak detector, it is necessary to characterize a peak detector. It is very necessary to characterize a peak detector to check for its non - linear properties as it comprises of non - linear components. To characterize a purchased peak detector was taken and its output at various input power levels for different frequencies was noted down. The peak detector taken was FLUKE 85RF II. The recorded data at some particular frequencies have been tabulated below.
TABLE I: Output of a peak detector for various input power levels at different frequencies.

Output rms voltage value

\begin{tabular}{|r|r|r|r|r|r|}
\hline Power $(\mathrm{dBm})$ & $\mathrm{f}=1 \mathrm{MHz}$ & $\mathrm{f}=15 \mathrm{MHz}$ & $\mathrm{f}=30 \mathrm{MHz}$ & $\mathrm{f}=100 \mathrm{MHz}$ & $\mathrm{f}=250 \mathrm{MHz}$ \\
\hline-30 & $4.3 \mathrm{mV}$ & $3.2 \mathrm{mV}$ & $3.2 \mathrm{mV}$ & $2.8 \mathrm{mV}$ & $2.7 \mathrm{mV}$ \\
\hline-20 & $34 \mathrm{mV}$ & $33.1 \mathrm{mV}$ & $30.3 \mathrm{mV}$ & $19.3 \mathrm{mV}$ & $19.7 \mathrm{mV}$ \\
\hline-10 & $166.9 \mathrm{mV}$ & $161.6 \mathrm{mV}$ & $153.6 \mathrm{mV}$ & $106.9 \mathrm{mV}$ & $110 \mathrm{mV}$ \\
\hline 0 & $0.488 \mathrm{~V}$ & $0.527 \mathrm{~V}$ & $0.507 \mathrm{~V}$ & $0.3679 \mathrm{~V}$ & $395.7 \mathrm{mV}$ \\
\hline 5 & $0.933 \mathrm{~V}$ & $0.922 \mathrm{~V}$ & $0.908 \mathrm{~V}$ & $0.637 \mathrm{~V}$ & $0.680 \mathrm{~V}$ \\
\hline 10 & $1.633 \mathrm{~V}$ & $1.747 \mathrm{~V}$ & $1.581 \mathrm{~V}$ & $1.0977 \mathrm{~V}$ & $1.188 \mathrm{~V}$ \\
\hline 15 & $2.446 \mathrm{~V}$ & $2.522 \mathrm{~V}$ & $2.506 \mathrm{~V}$ & $1.977 \mathrm{~V}$ & $2.042 \mathrm{~V}$ \\
\hline
\end{tabular}

From the above Table I, we plot the graph Output Voltage Vs Input Voltage. The plots have been shown below. From these plots, the linearity of the peak detector is understood.

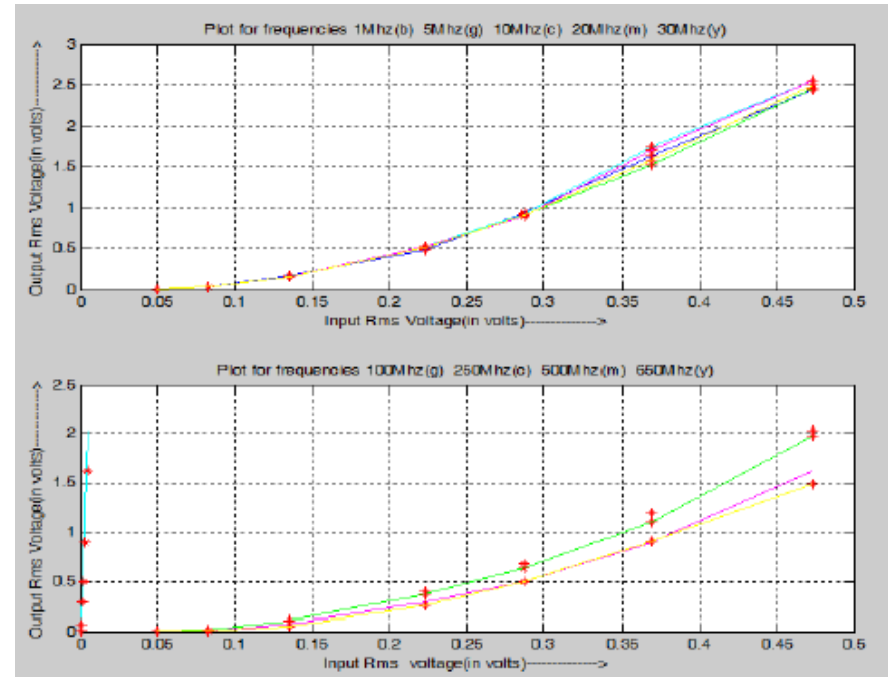

Fig 5. The plot of Peak Detector output Vs Peak Detector input

From Fig 5. it is clear that the peak detector does not respond well to inputs below 0.1 volts due to a turn-on voltage of the diodes. This drawback can be removed by externally biasing the diode circuit.

Now the step response of the peak detector is considered. A pulse is fed to the peak detector and the output is observed. The following figure shows the output of the peak detector. From the plot, it is observed that the peak detector requires a finite time to attain the maximum value when a pulse is applied in the input. This is due to the presence of the capacitor in the circuit. The capacitor requires a finite time to both charge and discharge.

Due to using a peak detector for the task at hand would not yield satisfactory results. Hence we change the direction of approaching the solution to the given problem and instead of detecting the amplitude of the change, the detection of a more fundamental and complex quantity of the signal, called phase, is carried out. This measured quantity is now used as a feedback to control the frequency shift and thus helps in frequency tracking. 


\section{METHODOLOGY}

In the above sections why a peak detector cannot be used for frequency tracking of the cavity has been explained. Now instead of using the peak detector, the phase detector has been used to solve the given problem. The detected phase is used to control the frequency shift of the resonant frequency to make the amplitude variation due to the vibrations minimum.

\section{A. Designing Of Frequency Tracking System}

The whole design of this system has been carried out in steps. The following block diagram depicts the whole system. The two major steps in this design were:

- Design of the phase detector.

Here a mixer followed by a low pass filter [4] is being used as a phase detector. As shown in Fig 6. the mixer receives the amplified cavity output at its RF port, whereas it also receives the reference signal from the signal generator at it's LO port. Now suppose due to the presence of the static or dynamic vibration on the cavity, the resonant frequency deviates from its original point. This would cause a phase change between the input and output signals of the RF cavity.

Let the generated signal be represented as

$x(t)=\cos (2 * p i * f r * t)$

then the output of the RF cavity would be

$s(t)=\cos (2 * p i * f r * t+p)$

where $p$ is the phase introduced due to the vibrations.

The mixer output would be

$I F=\cos (p)+\cos \left(4^{*} p i^{*} f r^{*} t+p\right)$

After passing this signal through a low pass filter with a cutoff frequency lower than or equal to $\mathrm{f}_{\mathrm{r}}$, we get an output that is directly proportional to the phase. When the cavity is at its natural state or the input signal has a frequency equal to the resonant frequency of the cavity then the phase i.e. the output of the low pass filter is zero. Now when the input signal frequency is increased beyond the resonant frequency of the cavity, we observed that the output of the low pass filter increases toward the positive side. The vice-versa is true for the condition when we decrease the input signal frequency.

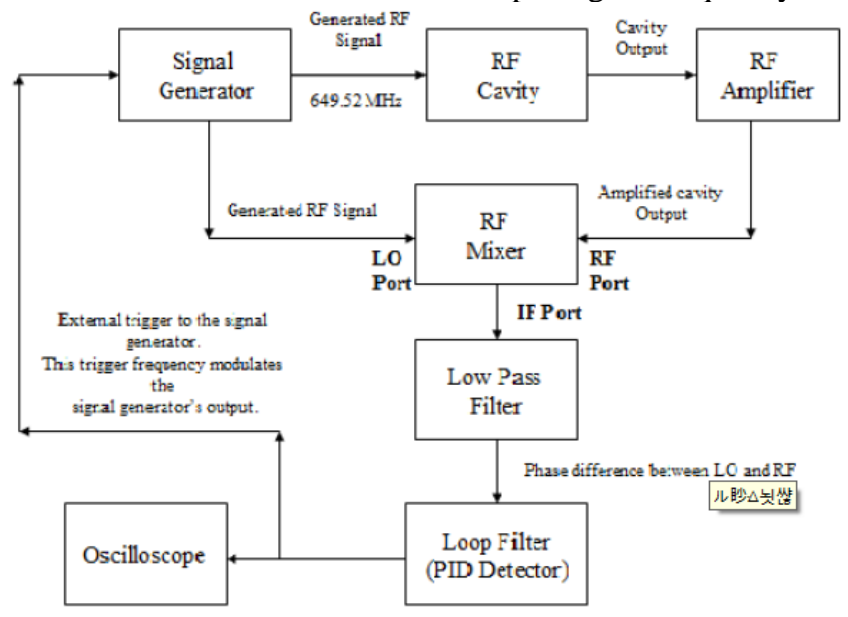

Fig 6. Block diagram of the frequency tracking system

Thus, a graph is plotted for phase vs. frequency for the phase detector.

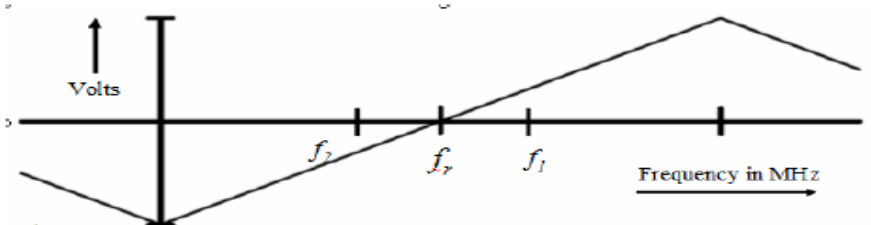

Fig 7. The output of the phase detector

where fr is the resonant frequency of the cavity. $\mathrm{f}_{1}$ is the frequency greater than the resonant frequency. $\mathrm{f}_{2}$ is the frequency smaller than the resonant frequency.

Fig 7. is true for the condition when static or dynamic vibrations are applied to the cavity as they change the resonant frequency of the cavity. Now for an increase in the resonant frequency, from the figure, it is observed that the output of the phase detector is positive. It is required to decrease the frequency; this is possible only by changing the sign of the external trigger voltage to the signal generator. This task is carried out by a loop filter.

- Design of loop filter

As discussed above the output of the phase detector needs to sign reversed before it can be applied to the signal generator as an external trigger. It is also needed to apply a certain gain to the output of the phase detector. The following circuit is designed (Fig 8.) that was chosen to function as a loop filter [7] for the given task.

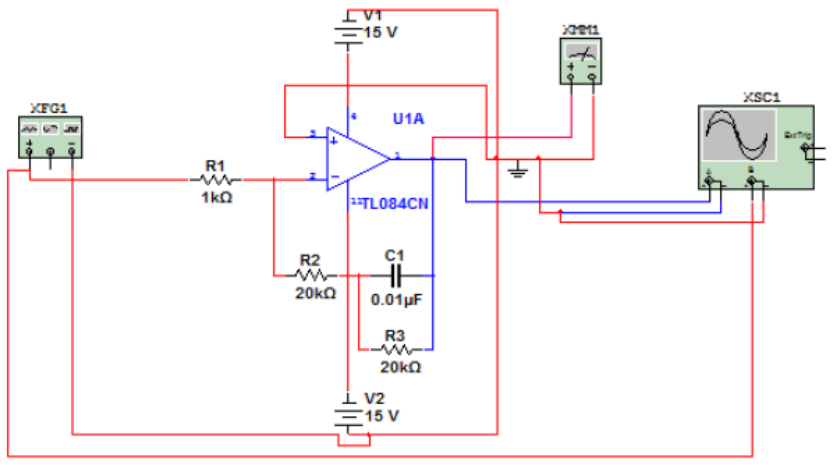

Fig 8. Circuit of the loop filter

This design gives a good result for both dynamic vibrations and static vibrations to the structure of the cavity.

\section{EXPERIMENTAL RESULTS}

The loop filter provides a gain of $(\mathrm{R} 2 / \mathrm{R} 1)$ to the short time vibrations. Generally, dynamic vibrations have very low dynamic range but in the frequency domain, they lie in the range of a few hundreds of $\mathrm{Hz}$ to $1 \mathrm{KHz}$. Thus this proportional term provides a sufficient gain to these vibrations. In the case of static vibrations such as compression, the integral action of the circuit comes into action. Static vibrations are generally of the range lower than $100 \mathrm{~Hz}$, thus the integrator provides stable steady-state performance for the system. It provides a gain of (R3/R1). The capacitor here charges with a time constant of $\mathrm{R} 2 * \mathrm{C}$. The step response of the circuit is shown below in Fig 9. 


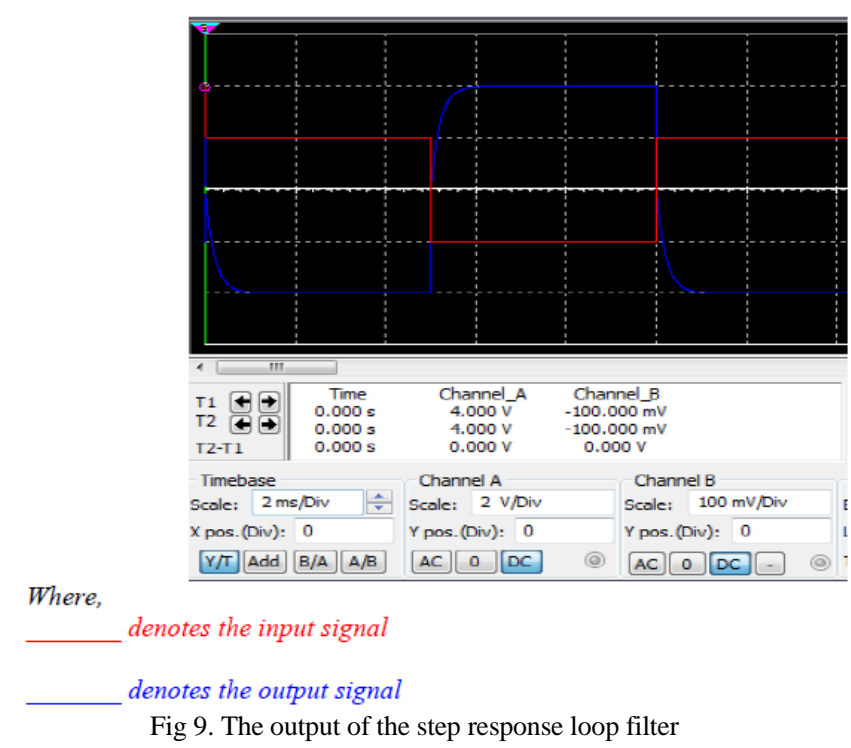

After the design of the loop filter and the phase detector, the circuit is connected as per the block diagram. This is a PLL [5] but instead of using a VCO, a signal generator was used. There were two reasons for the same. Firstly, a VCO has a fixed voltage sensitivity, thus if it was used then the circuit would have been application-specific. The signal generator provides an edge over the VCO as the voltage sensitivity can be varied as per the convenience. Secondly, a VCO is needed to be biased first to achieve the desired frequency at the beginning. This would increase the complexity of the circuit. Thus, a signal generator was used. The output of the phase detector was fed to the loop filter, which in turn changed the sign of the input signal and applied a gain to it. The output signal was fed to the signal generator as an external trigger. The option for frequency modulation was enabled along with the external trigger switch turned on. The voltage sensitivity of the generator was set to $40 \mathrm{KHz} / \mathrm{Volts}$. This value of voltage sensitivity was chosen because a stable output with no oscillations was obtained. An increase in the voltage sensitivity more than the specified value made the output to oscillate at $75 \mathrm{KHz}$. The frequency input to the RF cavity was also set in such a way that the output of the phase detector was zero. The range of frequency over which the output was not affected by the dynamic or static vibration was calculated. The values are given below.

1. Sensitivity $=40 \mathrm{KHz} /$ Volt.

2. The frequency at which the system was biased = $645.875 \mathrm{MHz}$

3. Range of frequency over which the $\mathrm{o} / \mathrm{p}$ was stable $=$ $645.850-645.90 \mathrm{MHz}$

4. The resonant frequency of the cavity $=645.892$ $\mathrm{MHz}$

It should be noted that the resonant frequency of the cavity and the frequency at which the system was biased are not the same. This was done to make the initial output of the phase detector to be zero, as at resonant frequency the phase detector output was not zero due to the presence of a dc offset. This also adds one complexity to the circuit. The phase detector output changes non-linearly with the increase of frequency. Though the disadvantage, the present biased frequency holds more advantages. Thus, this frequency has opted.

\section{CONCLUSION AND FUTURE WORKS}

In this research, a frequency tracking system for an elliptical RF cavity was successfully designed and tested. The concepts of basic phase-locked systems were employed to design the desired system. The loop filter, an important aspect of the phase-locked loop, was designed in a manner to work within a certain range of frequencies. But it lacks to work in full efficiency at the resonant frequency of the RF cavity. The mixer employed in the design of the system is a second-order non - linear system. This greatly affects the output response of the system.

In the future, it is needed to improve the design of the loop filter to make the response of the system better as well as to bias the system's operating frequency at the resonant frequency of the cavity.

\section{ACKNOWLEDGMENT}

I want to acknowledge my deep sense of gratitude to my research guide Professor Soumen Khatua who directed and guided me with his timely advice and constant inspiration which eased the task of my research work.

\section{REFERENCES}

[1] Magic tee: Structure Original Title: Waveguide by Jhon Deen Date uploaded on Jun 05, 2017

[2] P. K. Chaturvedi, Microwave, Radar \& RF Engineering, https://doi.org/10.1007/978-981-10-7965-8_3, Springer Nature Singapore Pte Ltd. 2018.

[3] Measurement of resonant frequency and quality factor of microwave resonators: Comparison of methods Journal of Applied Physics 84 3392 (1998); https://doi.org/10.1063/1.368498.Paul J. Petersan and Steven M. Anlagek

[4] Carter, B., \& Mancini, R. (2018). Active Filter Design Techniques. Op Amps for Everyone, 199-258. doi:10.1016/b978-0-R. Nicole, "Title of paper with the only first word capitalized," J. Name Stand. Abbrev., in press.

[5] General PLL Description: T. H. Lee, Chapter 15. Gray and Meyer, 10.4 Clock generation: B. Razavi, Design of Analog CMOS Integrated Circuits, Chapter 15, McGraw-Hill, 2001.

[6] Modern Digital and Analog Communication Systems by B.P. Lathi, $3^{\text {rd }}$ Edition, Oxford Publication.

[7] Chou, Y.-S., Chen, Y.-C., \& Chang, F.-R. (2008). Loop Filter Design for

Phase-Locked Loops with Guaranteed Lock-in Range. IFAC Proceedings Volumes, 41(2), 6410-6415. doi:10.3182/20080706-5$\mathrm{kr}$ -

1001.01081 .

[8] Analog Electronics Using Op-Amps by A. J. Peyton and V.Walsh, Cambridge University Press, 1993. 